\title{
Establishing a Conditional Signal for Assistance in Teenagers with Blindness
}

\author{
Muriel D. Saunders, Ana Carolina Sella, Dua Attri, and Richard R. Saunders \\ Life Span Institute, University of Kansas
}

\section{Abstract}

Five teenagers with severe intellectual impairments and no discernible communication skills were enrolled in training to teach a conditional request for assistance using a speech-generating device (SGD). All were either blind or severely visually impaired since birth. All learned to operate an adaptive switch to control sensory outcomes, next showed preferences among sensory outcomes, and then demonstrated the ability to use their switch to signal for assistance with an SGD when the sensory outcome was remotely disabled. During the signaling phase, or subsequent attempts to generalize its use outside the laboratory, 3 participants began vocalizing. Most notably, they began imitation of the word "song" or the word "help" emitted by the SGD. The potential role of causeand-effect training with adaptive switches is discussed.

A speech generating device (SGD) is an adaptive switch that activates a recorded message, an arrangement different from a switch that activates an object or a sensory event (e.g., music). Lancioni, O'Reilly, Cuvo, Singh, Sigafoos, and Didden (2007) conducted a review of research involving the teaching of requesting behavior to students with developmental disabilities using SGDs. The review indicated that in most research studies, preferred items were displayed, and one or more SGDs were within reach. The research participants were taught to press an SGD that produced a recorded message of a single word or phrase followed by the delivery of an item. Most study results indicated that training time to learn operation of the SGD was very short. Sigafoos, Didden, and O'Reilly (2003), for example, taught 3 young children with developmental disabilities to request preferred items using an SGD with the message, "I want more." All 3 participants learned to press their SGD to request within 20 minutes.

What was typically not tested in studies of SGDs is whether research participants learned when not to close the SGD. That is, did the participants learn to stay away from their SGD when preferred items had already been given or were not available? In educational practice, SGDs may fall out of use if the user does not signal with a message in a timely manner; that is, when relevant to do so and not to do so when not relevant. One study that described the training of a conditional request response was Sigafoos (1998). A child with autism was taught to point to a "want" symbol when a preferred item was out of reach and to reach for the item when it was close by.

\section{(C) 2013 Elsevier Ltd. All rights reserved.}

Corresponding Author: Richard R. Saunders Life Span Institute, University of Kansas, 1052 Dole Bldg., 1000 Sunnyside Ave. Lawrence, KS 66045 rrsaun@ku.edu (785) 864-1618 or cell: (913) 579-6043.

Publisher's Disclaimer: This is a PDF file of an unedited manuscript that has been accepted for publication. As a service to our customers we are providing this early version of the manuscript. The manuscript will undergo copyediting, typesetting, and review of the resulting proof before it is published in its final citable form. Please note that during the production process errors may be discovered which could affect the content, and all legal disclaimers that apply to the journal pertain. 
A few studies have reported increases in vocalizations following SGD training. Parsons and La Sorte (1993), for example, reported increases in spontaneous utterances in 6 young children with autism when the children had access to SGDs. All of the children had shown the ability to speak, but their words were not always used in a functional manner. Sigafoos et al. (2003) reported that one of their research subjects, a 3-year old with blindness and intellectual disability, began using words after learning to request using an SGD.

In the study described below, 5 adolescents with blindness or low vision and intellectual impairments were taught to signal for assistance. The aims of the study were 1) to identify a preferred form of sensory feedback, 2) establish control of the feedback consisting of closing an adaptive switch to activate feedback and staying away from the switch when feedback was already activated, 3) establish a reliable signal response using an SGD when the feedback was not available.

\section{Method}

\section{Participants}

Three female and two male participants from a larger group of participants are reported: Sue (female, age $20 \mathrm{yrs}$ ), Joe (male, age 14yrs), Ellen (female, age $15 \mathrm{yrs}$ ), Ron (male, age 11 $\mathrm{yrs}$ ), and Rachel (female, age $14 \mathrm{yrs}$ ). Sue had very limited vision in one eye. The others were blind from birth. All participants attended a school that specialized in educating students with low vision or blindness and significant cognitive delays with a functionally based curriculum. None of the participants had extensive experience with an adaptive switch, and none had access to switches immediately prior to the beginning of this study. Teachers and caregivers reported that none of the participants demonstrated functional verbal or symbolic communication abilities or imitated words or sounds. None of the participants had goals in their Individualized Educational Plan for increasing vocal imitation or functional verbalizations. All of the participants could be described as passive in daily activities; that is, they rarely initiated activities and often waited for others to prompt them throughout daily routines. The exception was Ron, who engaged in high rates of self-injury and aggression that were usually provoked in demand situations.

\section{Test of Communication Abilities}

A series of 10 scripted interactions designed to promote communication opportunities were conducted by the first author prior to the beginning of the study. Each script was designed to evoke requests for assistance to activate a novel object. During each script, an SGD was positioned near the participant. The SGD had the recording, "I need help" that played when the switch was closed. The child's hand was directed toward the SGD to indicate its presence. Objects were introduced that produced either auditory, visual, or tactile feedback or a combination of events. After 1-2 minutes of mutual participant-experimenter engagement with an object, the item was withdrawn or de-activated for 10-20 seconds. Any communicative responses by the participant were recorded during the 10-20 second window and responded to by acknowledging the response and sharing the object again. The window ended when the object was either re-introduced or put away and another object was presented. A total of 5 objects were presented twice. All scripts were videotaped and scored at a later time by a graduate student using the Complexity of Communication Scale (CCS) (Brady et al., 2012). The student was unaware of the procedures or results related to the adaptive switch or SGD. Inter-observer agreement checks were conducted on 3 of the 10 CCS tests.

The CCS is comprised of an ordinal scale with 11 levels. Level 0 is No Response and Level 1 is Alerting. Levels 2-5 are perlocutionary (pre-intentional), Level 6-9 are illocutionary 
(intentional), and Levels 10-11 are beginning locutionary (symbolic) communication development (Wetherby, Warren \& Reichle, 1998). Activations of the SGD would have been scored as Level 2 if the SGD was closed repeatedly throughout the running of the script, or at Level 3 if the switch was closed only during the window of time when the object was withdrawn or de-activated. However, none of the participants activated the SGD during scripts. Rachel scored at the Intentional Non-Symbolic level by reaching toward or touching an object, or vocalizing while interacting with an object. She picked up objects and moved them toward the experimenter. All other participants scored at the Pre-Intentional level by vocalizing or gesturing, but without interaction with the objects or SGD.

\section{Setting, Equipment, and Set-Up}

Each participant was seen individually once per day, 5 days a week for 20 minutes. Sessions were conducted by a para educator, hereafter referred to as the para, in a small room located near classrooms. The room contained a small table and two chairs. The participant sat in a chair across the table from the para. A Big Red ${ }^{\circledR}$ switch that was attached to a 9" $\times$ 9" board was strapped in a fixed position on the table top in front of the participant. The switch was connected to an adapted leisure device. Leisure devices included iPods, ${ }^{\circledR}$ vibration tubes, and mechanical toys. All devices were purchased commercially through specialty catalogs or local stores. The iPod was attached to an adaptive interface, described by Saunders, Questad, Cullinan, and Saunders (2011) that allowed music to be heard as long as the switch was closed. When the switch was released, music continued to play but could not be heard through the participant's earphones or speaker. The iPod® had a screen that allowed music genres to be displayed. Thus, music of a particular type could be selected by the para, instead of playing random music selections. Vibratory stimulation could be arranged to occur in several positions, such as behind the neck or on the legs.

In addition to the switch and leisure devices, a data collection interface was used to automatically record session length, cumulative switch closure duration, and a count of switch closures. Simple arithmetic procedures were used to calculate the relative proportion of each test session that each device operated.

The interface also permitted the choice of three contingency options. The first option was that a switch closure produced activation of a leisure device (for as long as the switch remained closed by the participant). The second was that a switch closure produced deactivation of the device; that is, the device was activated all of the time until the switch was closed (and remained deactivated for as long as the switch remained closed). In the third option, the interface permitted concurrent contingencies for two devices. In the concurrent contingency, a switch closure simultaneously deactivated a currently operating device and activated a second device. When the switch was opened (i.e., pressure was removed), activation of the second device ended simultaneous with the re-activation of the first device.

\section{Procedural Conditions}

To begin, participants were given the opportunity to use their switch to activate a variety of leisure devices. During this familiarization or practice phase, switch-closure demonstrations in the form of physical prompts were provided a) to indicate the position of the switch and b) to show the outcomes of a switch closures. Familiarization was discontinued when duration of device activation was at least $20 \%$ of the session for 2 consecutive sessions. Devices that the student activated longest were selected as the items for use for the remainder of the study. Each participant required 2-4 sessions to learn to close the switch independently, except Ron who required 22 sessions. Ron resisted sitting in a chair, and required frequent blocking of self-injury. 
Familiarization was followed by a series of test conditions to establish that each participant could a) respond differentially across two switch contingencies, in blocks of sessions, with one or more device outcomes (e.g., music), b) show this same differential responding when the contingencies changed daily, and c) show preferences across device outcomes. The sessions across these test conditions provided opportunities for the strengthening of switch use and contingency discrimination, but they were not designed as "teaching" conditions, as no prompts or instructions were provided.

The next condition was also designed as a test; in this case, a test of whether the participant's experience in the preceding conditions would serve as a sufficient prerequisite for the generalization of switch closure for signaling for assistance. The final test probed whether requesting assistance with a sensory device would generalize to signaling for assistance in simple tasks (e.g., opening a door to a playroom).

First test for differential responding-The test was comprised of 6 sessions of the activation phase followed by 3 sessions of deactivation, first with one device, then two to four additional devices. This test was conducted to compare performance and learning across devices. A strong indication of control was indicated if the percent of time closing the switch was less in the deactivation phase than in the corresponding activation phase.

Second test of differential responding-In this test, two devices were assessed that had produced strong indications of learning or contingency discrimination in the preceding test. The test included one session of activation followed by one session of deactivation for a total of 6 sessions with one device. Then the alternating sessions were repeated with a second device. The purpose of the second test was to determine whether the participant could change strategies for device control across sessions. Control was inferred if the percent of time closing the switch was longer in activation phases than deactivation phases for 5 out of 6 activation-deactivation pairings. That result would be expected if the device activated produced reinforcing stimuli.

Preference test-The purpose of this test was to determine which of two devices was the more preferred. Across 6 sessions the two contingencies-activation and deactivationwere alternated across the two devices across sessions. That is, in one session, Device 1 would be connected to the activation option and Device 2 would be connected in the deactivation option. In the next session, the setup was reversed. In the Preference Test, a device was inferred as the more preferred if the participant caused more output from that device than the other device in most sessions during the test, regardless of the contingency in effect for each outcome. Before the beginning of each session, the participant was prompted one time to close the switch and then release it, thus experiencing both outcomes. Otherwise, no prompts were provided during sessions. A device was inferred to be preferred if it was activated more than the other device in 5 out of 6 sessions.

Signaling for assistance-In this condition, the device identified as preferred in the Preference Test was connected to the interface in the deactivation option. After several minutes the switch was disconnected from the device surreptitiously using a switch that rechanneled the flow of electricity to an SGD. If the participant closed the switch, the SGD played the message, "Help me." Then the para responded immediately by saying, "I will help you." Next, the flow of electricity was rechanneled back to the leisure device. If a participant had learned to control a device in activation and deactivation phases, then it would be expected he/she would close the switch when the device was turned off surreptitiously. Responses were scored as correct if switch closure occurred within 3 minutes following a surreptitious disconnection and no closures occurred again until the 
next disconnection. Criterion performance was met when correct responses averaged $80 \%$ for 30 consecutive opportunities (disconnections).

Generalization of signaling for assistance with other tasks-A small SGD (VoiSec) was strapped on the participant's wrist with the recorded message, "Help me." The participant was then taken outside of the research room and given 4-8 opportunities to use their SGD to ask for help during a 20 min period. An opportunity began when an instruction was given that could not be followed by the participant without assistance. For example, a participant was asked to go to the door, with the expectation of opening the door and going out (a difficult task unless assistance was provided). Initially, the participant was prompted to close his/her SGD. Prompts were faded until the participant was asking for help with the SGD at appropriate times. Then instructions that could be followed without assistance (e.g., walk with me) were intermixed with those that could not be followed. The number of independent and prompted responses was recorded each session. Again, criterion was set at an average of $80 \%$ independent responses across 30 opportunities.

\section{Results}

The accuracy of the data collection interface was tested at the beginning of each session by the research assistant. The switch connected to the interface was closed several times and the count on the interface was observed after each closure. The research assistant also noted whether the clocks (session duration and response duration) were running. The primary investigator observed a research session with every participant every two months across the period of the study to confirm that the research protocol was followed. The protocol was an electronic arrangement of switches and devices and not a set of behaviors by the research assistant, except for remote disconnections. An investigator also attended one session at the criterion level of the Signaling and Generalization phases and determined that procedures were being following, and that data were being collected correctly.

Inter-observer reliability was calculated for CCS tests by comparing interobserver scores for each of the 10 scripts that comprised each test. Two observers agreed on $86.6 \%$ of the scores.

\section{Results for $\mathbf{1}^{\text {st }}$ and $2^{\text {nd }}$ tests of differential responding}

Figure 1 shows the results of the first test for all 5 participants. A strong indication of control was indicated if all of the data points in the deactivation phase were lower than 3 out of the final 4 data points in the corresponding activation phase. Each participant showed the indicators for control for at least 2 devices. Sue and Joe showed higher rates of switch closures in the activation than deactivation contingency but switch use did not occur for more than $50 \%$ of the session in either condition. Ellen maintained almost continuous activation when the controlled outcome was stories. Ron and Rachel maintained activation above $90 \%$ across sessions regardless of the contingency.

Figure 2 shows the results of the second test of control for all participants. Sue showed the ability to control both sound-visual devices and music, although she controlled sound-visual devices by maintaining them in the off position. This suggests not only that she could control devices with her switch, but that she had a clear aversion for sound-visual devices. Joe showed little control in either contingency. Ellen maintained control when stories were the outcome under switch control. Ron and Rachel showed the ability to maintain almost constant activation across activation and deactivation contingencies. 


\section{Results of preference tests}

Figure 3 shows the results of the preference test. Each participant showed higher activation time with one device over another across sessions. All of the participants except Ellen had at least one session when they activated the apparently less preferred device more of the time. Ellen changed what device was activated predominantly after the second session. Joe maintained both devices around 50\% of the time, which may indicate either weak control or enjoyment of both outcomes.

\section{Results of requesting for assistance with a sensory device}

In Figure 4, the data to the left, under the title, "Signaling," show the number of correct SGD responses in bundles of 10 opportunities in the Signaling phase for all 5 participants. A correct response was a press to the SGD within 3 minutes of a disruption to the preferred device and no presses to the SGD when the preferred device was re-activated. All participants met criterion for signaling, although Sue and Joe required more training trials than the others.

\section{Results of generalization of signaling for assistance with other tasks}

Figure 4, data to the right, under the label, "Generalization of Signaling," show the number of correct SGD responses in bundles of 10 opportunities in situations outside the research room when assistance was needed for Sue, Joe, Ellen, and Rachel. Sue's generalization data indicate correct signaling at the beginning of training, but after 220 opportunities to use her SGD, she had not met criterion. Ellen consistently asked for some activities with her SGD, such as help in finding the playground, and in finding the swing, however, she did request consistently across activities. Joe and Rachel used their SGDs in situations outside the research area at criterion level.

Ron was not tested for generalization with the SGD because he began using words to ask for assistance. This result was not expected. Although it has been suggested that an SGD might foster word development in children that already use vocalizations for communication (Rhea, 1997), the experimenters did not expect any if the participants to show the ability to spontaneously emit or imitate words after exposure to SGD training. In screening students for this project, teachers were asked to nominate those who did not imitate or otherwise communicate needs. The scripts designed to engender communication abilities that were conducted at baseline did not produce spontaneous requesting by the participants and did not produce vocal imitation.

Ron began using verbalizations to indicate his needs before learning to signal. His first word was "song" which he said when he heard the voice of the para prior to the beginning of research sessions. Next, he learned to say, "Help" first, as an imitation to a verbal model and then spontaneously. In the 6 months after completing the tests for signaling, he learned to say the following words during specific routines: good morning, stop, finished, spin, jump, walk, clap, ball, take it off, sit down, and put it on.

Prompted by Ron's spontaneous verbalizations, attempts were made to promote verbalizations in the other participants. After the lengthy training for generalization, Ellen's procedures were changed to promote a spoken request response. She was given an instruction to go to the playground and she used her SGD to ask for help in getting there. Then the SGD was removed from her wrist, and she was given an instruction to find the merry-go-round. After a short pause, she was given a model to say the word, "Help." Instructions followed by the model "Help" were repeated for 45 minutes and Ellen finally imitated the word "Help". Within 6 weeks she was imitating most words. After more than 6 months of training, Ellen learned to label objects and to greet people using spoken words. 
Rachel generalized use of the SGD to other settings almost immediately. After prompting Ellen to request assistance using a spoken word, the same procedure was attempted with Rachel. She also imitated the word, "help." Within 6 months, she was imitating the following words: help, walk, pen, book, hi, bye, mama, papa, and ball. She also learned to label a variety of objects using tangible symbols and words. When asked to name an object, there was typically a 15-20 sec delay between asking the question, "What is this?" and her response.

The same procedures for prompting a spoken word were attempted with Sue and Joe. Sue never imitated vocally during training. She used her SGD inconsistently to request assistance, but when given an instruction, she began touching the para's hand or arm and moving it in the direction where help was needed. Thus, she learned to communicate her need for assistance more consistently using a gesture than with the SGD. After 6 months she continued to use the gesture when she needed help in classroom activities. Joe did not show any communicative gestures or vocalizations at the completion of training. After 6 months he continued to use his SGD in the classroom.

\section{Discussion}

The present procedures were designed to test for the development of independent control of leisure devices using an adaptive switch. The detection of such control (i.e., differential responding) then permitted testing for relative preference among devices. Identification of a preferred device strengthened the likelihood that the procedures in the signaling phase would engender signaling by the participant. A particularly important observation regarding these data is that items that produce significant control over responding individually may not show equal control when contrasted. That is, stimuli that may have considerable power as reinforcers, may be of considerably different relative strengths. Further, participants may show strong preferences among stimuli that appear to be equally weak reinforcers.

Overall, the procedures were simple, followed a step-by-step sequence, and incorporated principles of reinforcement. All five participants learned to request assistance in activation of the preferred device when it was turned off and to refrain from requesting restoration when it was already turned on. The results suggest that those with limited communication abilities can be taught to discriminate when to use an SGD appropriately when the task is broken down into a step-by-step format.

One can only speculate why some of these teenagers showed an increase in oral communication skills. Their blindness may have been a factor. Vision is the dominant sensory modality in the development of early language (Andersen, Dunlea, \& Kekelis, 1993; Bigelow, 2003; Preisler, 1991; Urwin, 1979). Indeed, one third of children with partial sight and two thirds of children with blindness have developmental disabilities (Batshaw, Pellegrino, \& Roizen, 2002). It may be difficult to determine exactly why so many children with blindness have developmental delays, however. Congenital blindness is often caused by prenatal infection, birth injury or a genetically determined condition (Blindness,

MassGeneral Hospital for Children). These conditions may produce cognitive problems as well, that in turn, produce delays in language development. Thus, it may be difficult to untangle the effects of blindness from other health and cognitive conditions present in those with blindness.

The staff at the school appeared to consider blindness to be the primary disability of the participants in this study. Ambulation and independence in daily routines were emphasized, and activities to prepare the teenagers for using Braille was the primary communication skill 
taught to students. The school district provided speech therapy for students that showed the necessary skills for oral language but none of these participants were chosen for this service.

Each of the participants presented challenges. Ron, for example, had little success in learning new skills in his classroom. He engaged in almost constant self-injury and other disruptive behaviors. Instruction of functional routines were limited to personal care, safety and well being. He spent most of his day lying in a beanbag chair and standing while listening to music. When music was provided contingent upon closing an adaptive switch, it was found to be a powerful reinforcer. Ron's data indicate he learned to control activation of music almost without error. The para's voice became a discriminative stimulus for going to listen to music. The para also consistently announced the activity by saying, "Let's hear songs" and his first clear word was "song."

Ron rapidly moved from imitation of words to functional manding. He now asks for favorite objects and activities as well as controlling activities through the use of the words "stop," "finished", or "help." He also gains attention from others by using the words of others in routines, such as "sit down" and "put it on," in something of a "say-do" manner. He also enjoys the attention provided when he imitates phrases spoken by others.

Ellen often responded to instruction by giggling, pushing and then leaving the area. She may have had the ability to imitate words in the past, but over the years she may have not been required to speak. When teachers heard her ask for assistance using the word, "help", they may have begun requiring more verbal imitations from her. She now gains attention from others using social comments as well as by imitating comments of others.

Rachel was extremely passive. When given a simple instruction, she would wait until she received assistance in completing the activity. Furthermore, she emitted no sounds. Her parents reported that until the study, she had never said the words "mama" and "papa." Further, her educational staff did not become aware that Rachel exhibited an unusually long latency between a question or model and her response until oral language was established in this study. Her potential for vocalizations may have been high from an early age, but they did not emerge as a function of frequently or always being preempted. Her overall passivity may have further discouraged any expectation of the acquisition of independent responding.

Our procedures also did not test whether the participants learned to close the switch to activate their leisure devices as a function of the training they received in this study, but it did verify that learning had occurred. Most switch programs indicate that learning occurred as a result of training by incorporating an A phase in which the switch does not control a leisure device, and then a B phase when the switch operates the device. We have found that a return to $\mathrm{A}$ conditions in an $\mathrm{ABAB}$ format disrupts later switch use in activation conditions; that is, response reduction by extinction can be difficult to overcome, and should be avoided with individuals with severe intellectual impairments.

Future research needs to be conducted on the use of SGDs. Other instructional procedures need to be developed for teaching conditional responding with SGDs that contain a variety of messages. These procedures need to be assessed for generality with children and adults with a variety of disabilities including blindness. Further, periodic testing of vocal or verbal imitation skills as well as other communicative behaviors should be conducted before and during SGD training.

What is the most significant contribution to the literature made by this study? Perhaps it is that simple, low-level operant teaching procedures can have an important role in a) verifying learning capabilities and $b$ ) identifying reinforce preferences in persons with significant developmental delays. In this study, while all participants demonstrated reinforcer 
preferences, Sue demonstrated a clear aversion to a particular sensory outcome, Rachel revealed a critical learning characteristic (delayed responding), and Ron's acquired control over music provided a potential intervention pathway through his self-injury. These results argue strongly for sustained, systematic, individualized assessments with operant procedures before children with severe delays are placed into "integrated" classrooms where their needs and skills will not likely be detected.

\section{Acknowledgments}

Support for this study was provided in part by grants HD018955 and HD02528 to the Schiefelbusch Institute for Life Span Studies from the Eunice Kennedy Shriver National Institute of Child Health and Human Development. The authors also are grateful to the staff of the Special Education School of the Junior Blind of America for their assistance and cooperation. In particular, we acknowledge Griselda Espinosa's dedication to our work and the children with whom she worked.

\section{References}

Andersen EE, Dunlea A, Kekelis LS. The impact of input: Language acquisition in the visually impaired. First Language. 1993; 13(1):23-50.

Batshaw, ML.; Pellegrino, MD.; Roizen, NJ. Children with Disabilities. 6th ed.. Paul H. Brookes; Baltimore: 2002. p. 158

Bigelow A. The development of joint attention. Development and Psychopathology. 2003; 15:259275. [PubMed: 12931827]

Brady NC, Fleming K, Thiemann-Bourque K, Olswang L, Dowden P, Saunders MD, Marquis J. Development of the Communication Complexity Scale. American Journal of Speech-Language Pathology. 2012; 21:16-28. [PubMed: 22049404]

Lancioni GE, O'Reilly MF, Cuvo AJ, Singh NN, Sigafoos J, Didden R. PECS and VOCAs to enable students with developmental disabilities to make requests: An overview of the literature. Research in Developmental Disabilities. 2007; 28:468-488. [PubMed: 16887326]

MassGeneral Hospital for Children. [July 19, 2011] Blindness. n.d. from http://www.massgeneral.org/ children/adolescenthealth/articles/aa_blindness.aspx

Parsons CL, La Sorte D. The effect of computers with synthesized speech and no speech on the spontaneous communication of children with autism. Australian Journal of Human Communication Disorder. 1993; 21:12-31.

Preisler G. Early patterns of interaction between blind infants and their sighted mothers. Child: Care, Health, and Development. 1991; 17:65-90.

Rhea P. Facilitating transitions in language development for children using AAC. Augmentative and Alternative Communication. 1997; 13:141-147.

Saunders MD, Quastad KA, Cullinan TB, Saunders RR. Adapted digital music players for individuals with severe impairments. Behavioral Interventions. 2011; 26(2):161-166.

Sigafoos J. Assessing conditional use of graphic mode requesting in a young boy with autism. Journal of Developmental and Physical Disabilities. 1998; 10:133-151.

Sigafoos J, Didden R, O'Reilly M. Effects of speech output on maintenance of requesting and frequency of vocalizations in three children with developmental disabilities. Augmentative and Alternative Communication. 2003; 19:37-47.

Urwin C. Preverbal communication and early language development in children with blindness. Papers and Reports on Child Language Development. 1979; 17:119-127.

Wetherby, A.; Warren, S.; Reichle, J., editors. Transitions in prelinguistic communication. Vol. 7. Brookes; Baltimore: 1998. p. 365-385. 


\section{Highlights}

- Cause and effect learning was demonstrated in children with blindness and no communication skill.

- In a single adaptive-switch method, preferences between sources of stimulation were observed.

- Participants learned to signal for assistance with stimulation using a speech generating device.

- Generalization of signaling was demonstrated outside the research setting.

- During generalization testing, oral or gestural communication arose in 4 of 5 participants. 

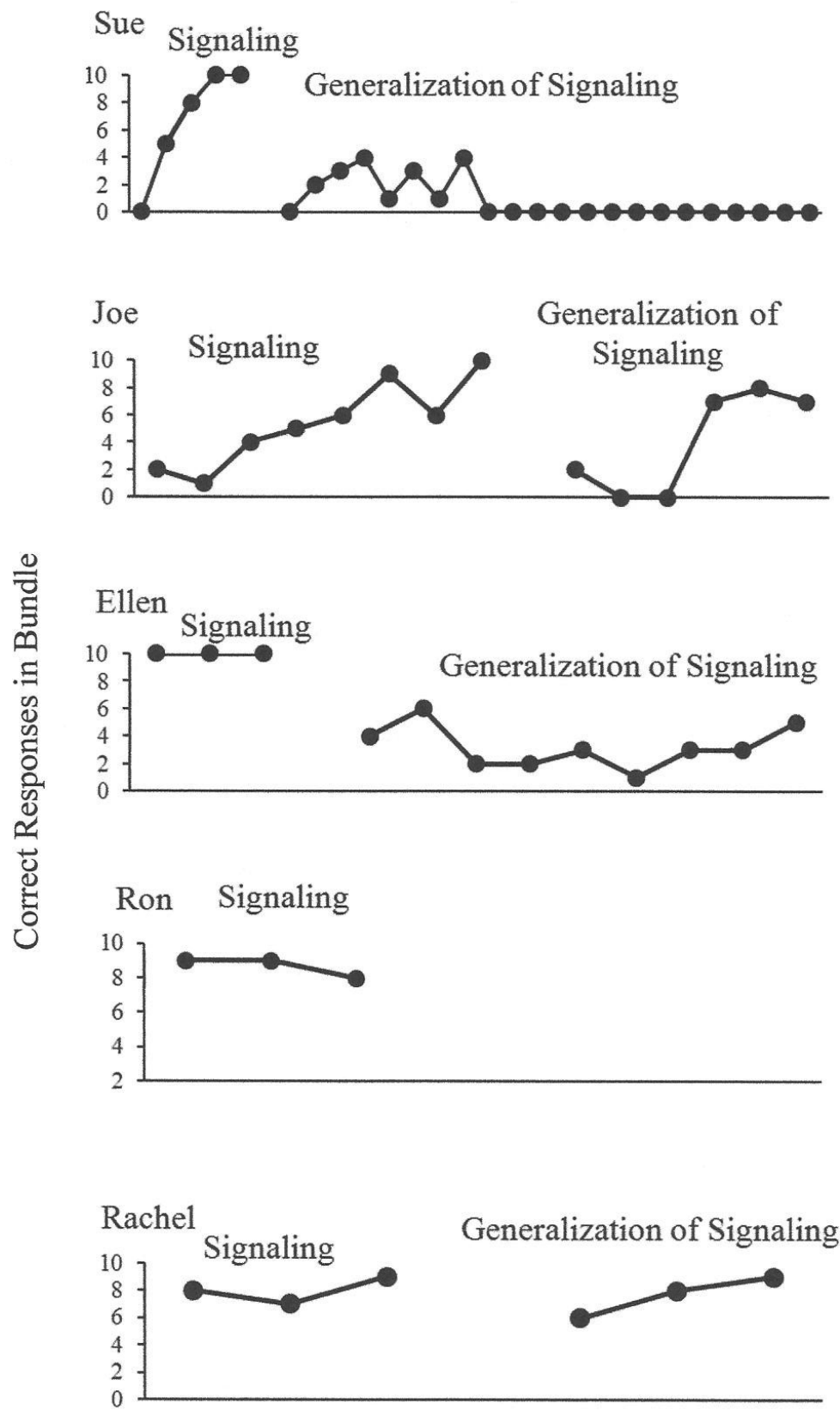

\section{0-Opportunity Bundle}

Figure 1.

Results of the $1^{\text {st }}$ Learning Test for each participant. The percent of time that the switch was closed are shown for 6 sessions of the activation condition (closure causes Device operation) and 3 sessions of the deactivation condition (D, closure cause Device inoperation). The data points labeled "A" are sessions in the activation condition and those labeled " $\mathrm{D}$ " are sessions in the deactivation condition. 
Sue
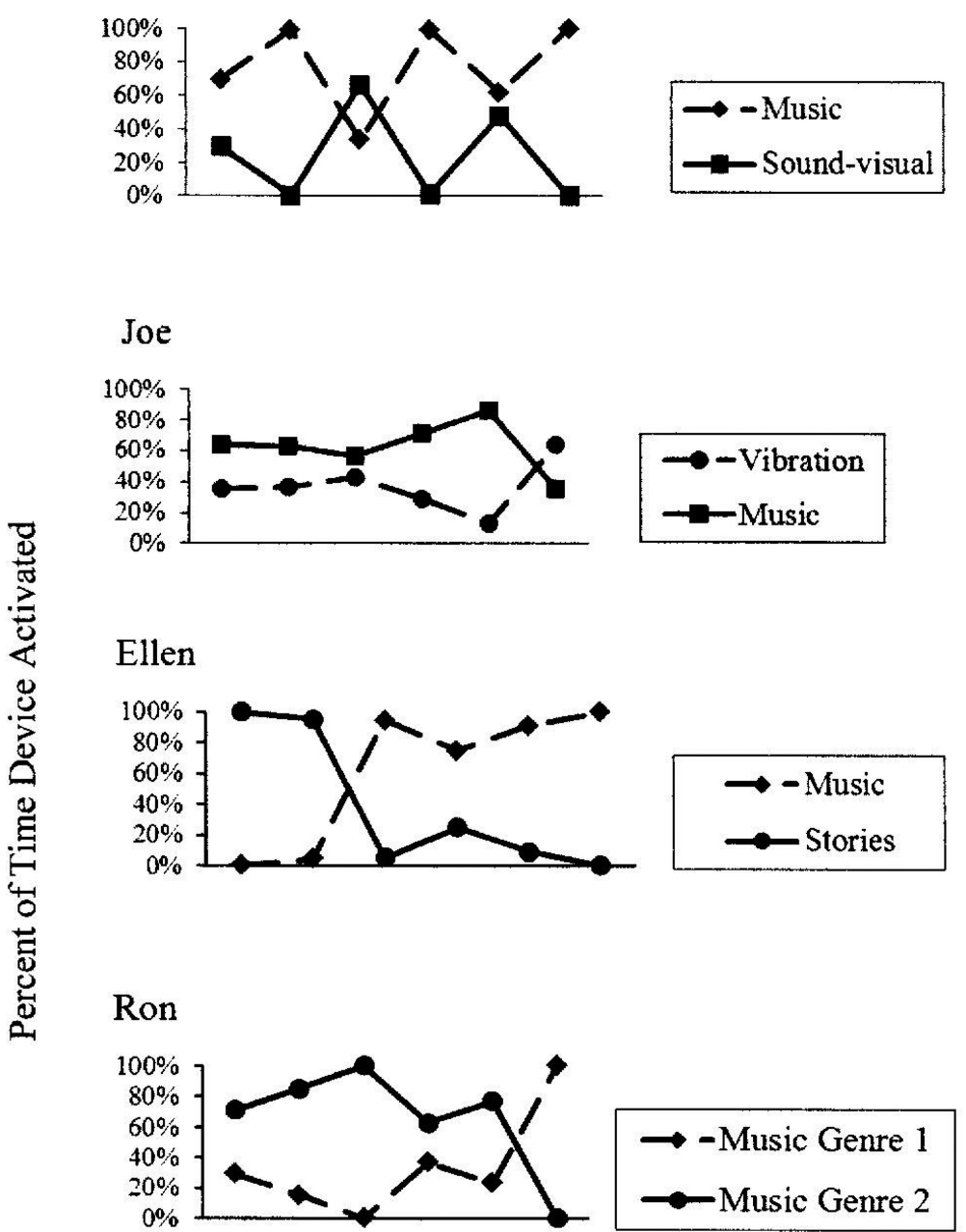

Rachel
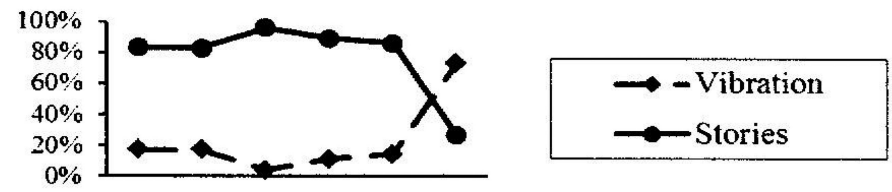

Sessions

Figure 2.

Results of the $2^{\text {nd }}$ Learning Test for each participant. Activation sessions were alternated with deactivation conditions. 

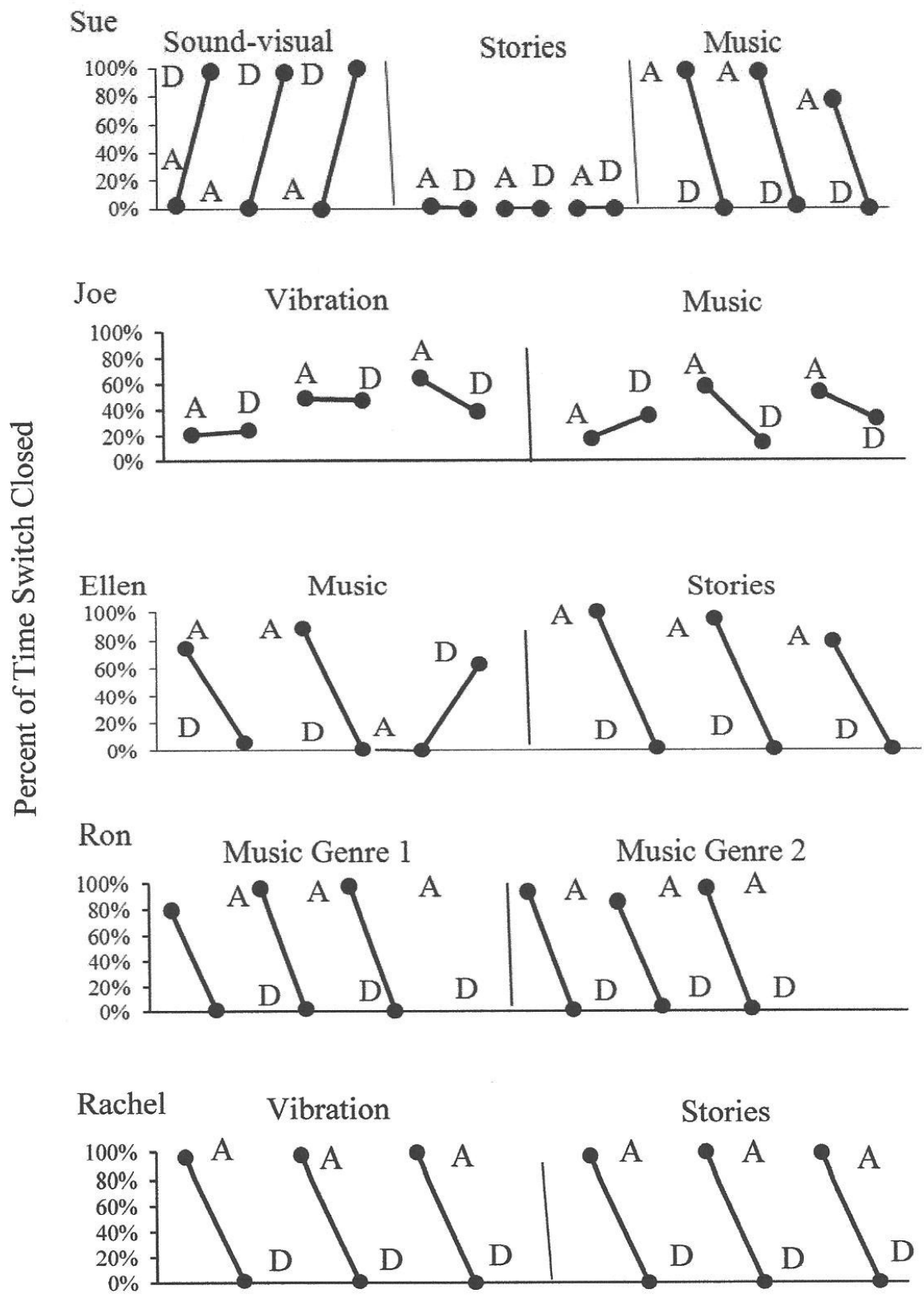

\section{Sessions}

Figure 3.

Results of the Preference Test for each participant, in a concurrent access arrangement with access contingencies-activation and deactivation -alternated across devices across sessions. 
Sue

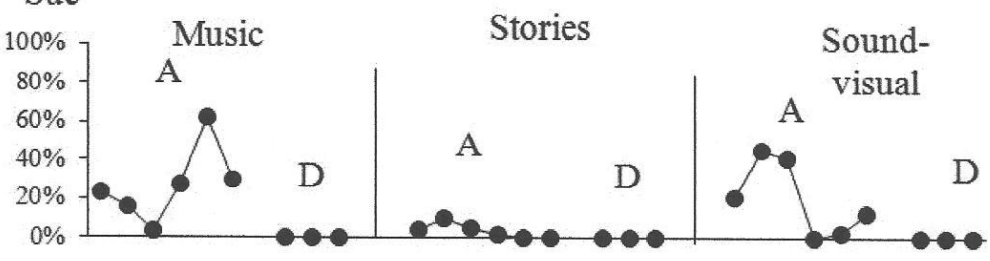

Joe

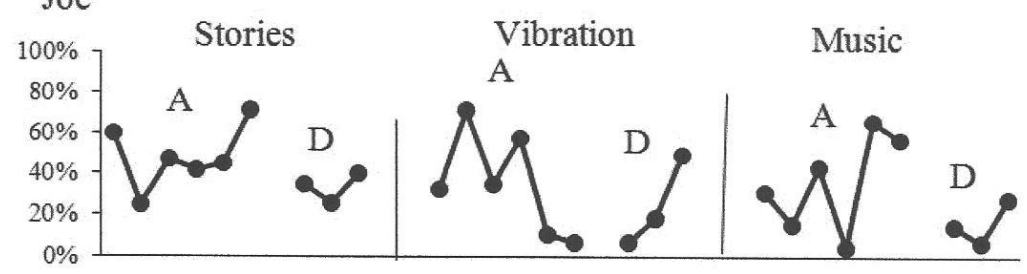

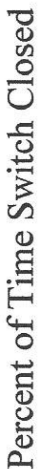

Ellen

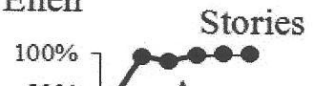

Vibration
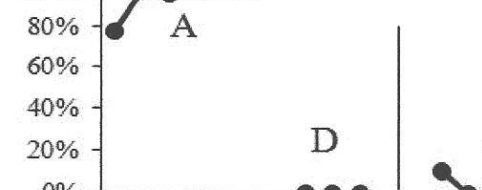

Ron

Ron Music Genre 1

$100 \%$ A

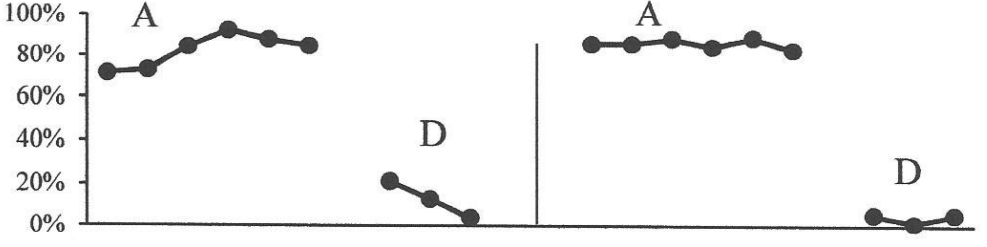

Rachel

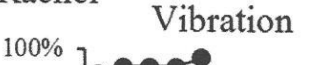

$\left.\begin{array}{r}100 \% \\ 80 \% \\ 60 \% \\ 40 \% \\ 20 \% \\ 0 \%\end{array}\right]$

A
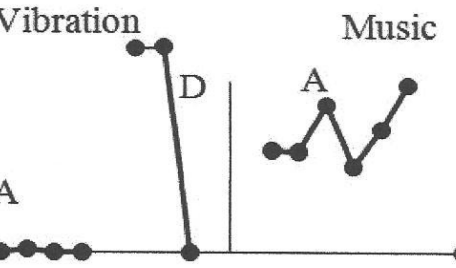

$\mathrm{D}$

D

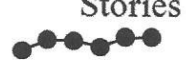

A

s
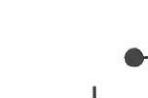

Music

A

Sessions

Figure 4.

Results of the phase to teach Signaling and Generalization of Signaling for Sue, Joe, Ellen, and Rachel. Shown to the left are percent correct reactions to disconnections-switch closure within $3 \mathrm{~min}$ of disconnection followed by no switch closures until the next disconnection--in the Signaling phase. Shown to the right are the percentages of opportunities the portable SGD was used unprompted within 3 min to request assistance. 